\title{
COMMENTARY
}

\section{Educational Utilization of Microsoft Powerpoint for Oral and Maxillofacial Cancer Presentations}

\author{
Francisco Samuel Rodrigues Carvalho ${ }^{1}$, Filipe Nobre Chaves $^{2}$, Eduardo Costa \\ Studart Soares ${ }^{1}$, Karuza Maria Alves Pereira ${ }^{2}$, Thyciana Rodrigues Ribeiro', \\ Cristiane Sa Roriz Fonteles ${ }^{1}$, Fabio Wildson Gurgel Costa ${ }^{1 *}$
}

\begin{abstract}
Electronic presentations have become useful tools for surgeons, other clinicians and patients, facilitating medical and legal support and scientific research. Microsoft ${ }^{\circledR}$ PowerPoint is by far and away the most commonly used computer-based presentation package. Setting up surgical clinical cases with PowerPoint makes it easy to register and follow patients for the purpose of discussion of treatment plan or scientific presentations. It facilitates communication between professionals, supervising clinical cases and teaching. It is often useful to create a template to standardize the presentation, offered by the software through the slide master. The purpose of this paper was to show a simple and practical method for creating a Microsoft ${ }^{\circledR}$ PowerPoint template for use in presentations concerning oral and maxillofacial cancer.
\end{abstract}

Keywords: PowerPoint presentations - audiovisual aids - computer-assisted learning - oral cancer

Asian Pac J Cancer Prev, 17 (4), 2337-2339

\section{Introduction}

In oral cavity, the oral squamous cell carcinoma is the most common maligantt entity, accounting for over $90 \%$ of the cases of diagnosed malignant tumors (Scully, 2005). The mortality rate regarding oral cancer is estimated at approximately 12,300 deaths per year, and the survival rate is simply $40 \%$ to $50 \%$ for diagnosed individuals and is closely related to the early disease perception time between and its diagnosis and treatment plan (Dantas et al., 2016). In the field of oral cancer, there are important researchers focusing on knowledge of oral cancer among adult dental subjects attending dental clinics (Razavi et al., 2015), which highlights the need of other studies involving the use of educational strategies for oral cancer evaluation.

In this context, the advent of digital photography allowed the photographic documentation to become routine in many services of oral and maxillofacial cancer. In addition, the acquisition and storage of photographic documentations has become easier, faster and with reduced costs. Setting up surgical clinical cases with Microsoft ${ }^{\circledR}$ PowerPoint may facilitate the photographic recording and the patient follow-up, aiming to its use in clinical sessions for treatment plan discussion or in scientific presentations. It facilitates communication between professionals, teaching and supervising of clinical cases. Cases may be set up individually, facilitating the treatment planning, sharing experiences involving clinical cases and new techniques, and exchanging digital information with other experts or residency programs (Hegarty, 1999).

This program has become popular due to the many advantages it offers, including the ease of making last minute changes and customization, the possibility of incorporating multimedia files, portability, and cost reductions (Regennitter, 2000). Clinical images may be enhanced through alignments, cropping, brightness/ contrast optimization, and color corrections, requiring a minimum of skills by users (Halazonetis, 2002).

Microsoft ${ }^{\circledR}$ PowerPoint has been described as an incorporation software. Images are easily produced, edited and stored without loss of quality or resolution. It is the state of art in presentation software, with the addition of clinical content (Hegarty, 1999).

In a presentation, the creation of a standardized template it is often useful. Microsoft ${ }^{\circledR}$ PowerPoint provides the Slide Master as a valuable tool. Although this tool is not shown in the presentation, it serves as a template the top slide in a hierarchy of slides that stores information about the theme and layout of a presentation, including the background, color, fonts, effects, placeholder sizes, and positioning. In addition, the Slide Master allows visually satisfactory results, optimizing time in long-duration presentations, since all changes can be performed at one time (Halazonetis, 1998).

The purpose of this paper was to show a simple and practical method for creating a Microsoft ${ }^{\circledR}$ PowerPoint 
template for use in oral and maxillofacial cancer and how to perform the insertion of multiple photos that standardized presentation, as to the comparative. The analysis of photographic records is remarkably important for surgeons and patients to assess the surgical outcomes, as well as or medical and legal support and scientific research.

\section{Practical Measures}

\section{Creating a Microsoft ${ }^{\circledR}$ PowerPoint template}

A suitable and visually attractive PorwerPoint presentation requires a general knowledge about the Slide Master functionality (Figure 1). It is possible to insert master slides (e.g. slides representing the preoperative period, imaging exams, and postoperative evaluation) with proper layouts. Besides the possibility of inserting several layouts in each Slide Master, there are a number of options that can be easily editable as described in the Figure 1. Additionally, the figure placeholders can be arranged to provide an adequate orientation of the image rotation arrows according to object position during the photographic record (Figure 2). For example, if a profile was photographed with the flash towards the left, the

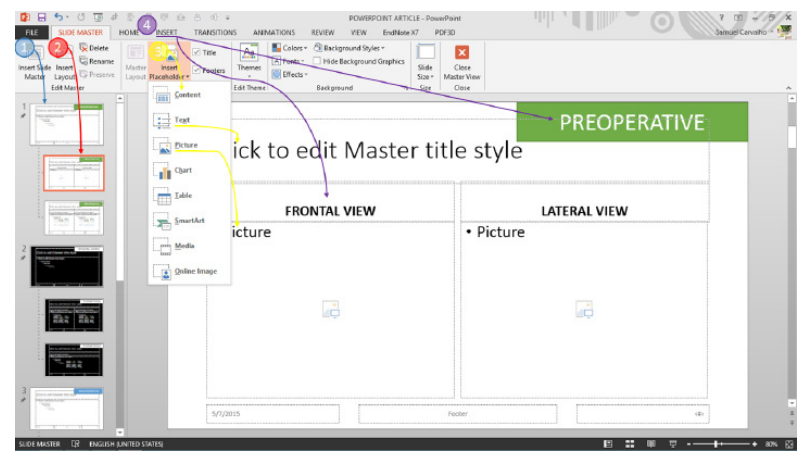

Figure 1. Slide Master Ribbon. 1) Insert Slide Master; 2) Insert layout; 3) Insert placeholder (aiming to insert editable objects during the making of the presentation outside the Slide Master); 4) Menu insert (aiming to insert objects, text boxes, figures to be repeated in the slide structure, not amenable to issue during the preparation of the presentation outside the Slide Master)

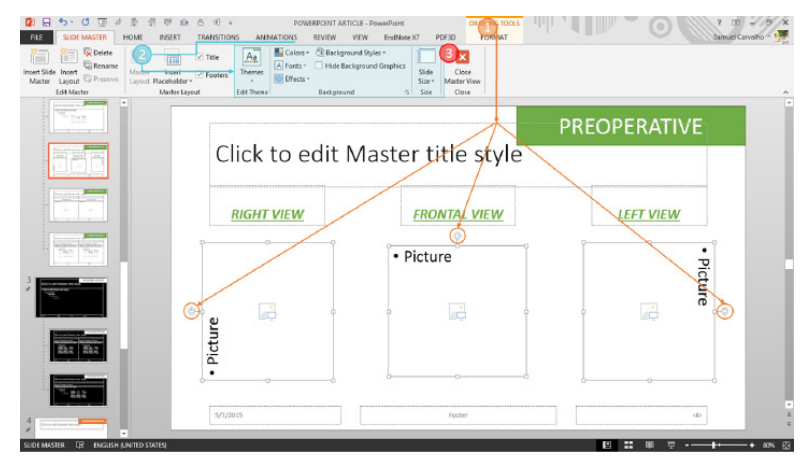

Figure 2. Final Adjustments of the Slide Master. 1) Drawing menu (it allows the image placeholders rotation to ensure the guidance of the insertion of photographs); 2) options to customize the theme presentation. 3) Close master view (it should be activated after the completion of all the changes). arrow above the placeholder should be pointed in the left. Likewise, in occlusion photos the arrow should point in the same direction as the flash: upwards for upper occlusion and downwards for lower occlusion. When photographs are taken in a standardized manner, no image rotation is required.

After creating a Slider Master and its layouts, it is possible to rename the slide and customize the theme

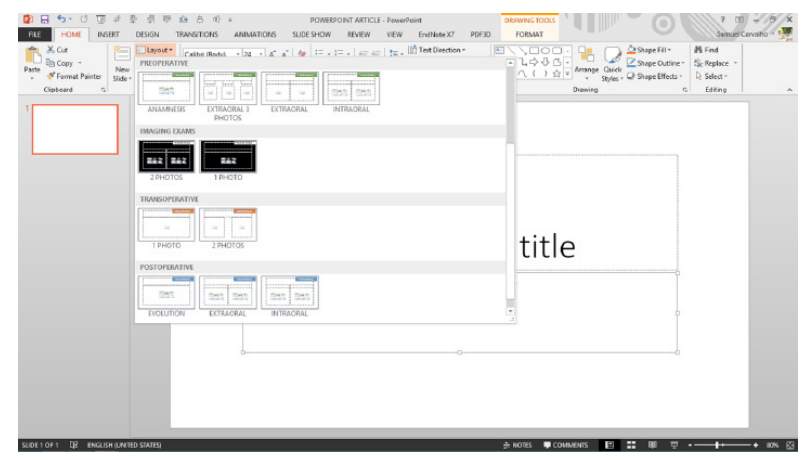

Figure 3. Representation of Both Customized Slide Masters (Preoperative and Postoperative) Showing Respective Layouts

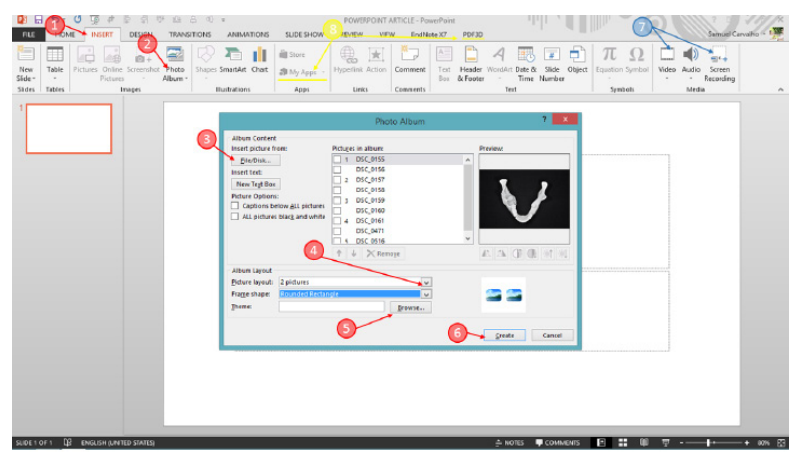

Figure 4. Inserting Multiple Images. 1) Insert; 2) Photo Album; 3) File/Disk; 4) Picture layout (determines the distribution of pictures on slides); 5) Browse (allows you to select a design template); 6) Create (create the presentation with all selected pictures; 7) Panel to insert other midia files; 8) My Apps (allows to import Apps).

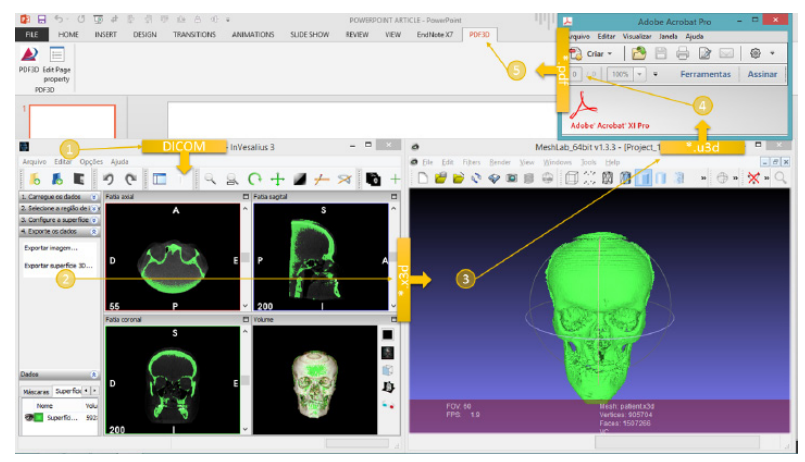

Figure 5. In-app functionality of rotation structures in a virtual 3-D space. 1) Import the DICOM data to a specific software; 2) Create a reformatting surface structure and export as *.x3d format; 3) Open this file in MeshLab software and export to *.u3d format; 4) Convert this file in a *.pdf format using Adobe Acrobat XI Pro; 5) After installing 3DPDF software, an icon on the ribbon will allow to attach the created PDF file, which offers a real time tridimensional handling. 
with appropriate colors, fonts, effects, background styles and size (Figure 2). Subsequently, as described in Figure 3 , these customized layouts will appear on the layout command of the home tab situated on the ribbon. However, to add this new design to the existing PowerPoint themes is necessary to save it as a PowerPoint Template (.potx) file.

\section{Inserting multiple photos or other medias files}

Microsoft PowerPoint offers the Photo Album as an interesting tool, which allow the inclusion of several images in a presentation. To insert a lot of pictures into the presentation, see the first 6 steps described in the Figure 4.

In addition, a versatile option available in PowerPoint is the incorporation of other media files such as videos and screen-recorded images (Figure 4). There is the possibility of incorporating exported videos from 2-D and 3-D image viewers, as well as it is possible to attach a video from a recorded screen while manipulating a 3-D reconstructed image in a specific software (example: InVesalius 3.0).

2-D images of 3-D representations are a poor substitute for the in-app functionality of rotationg structures in a virtual 3-D space. As an alternative to this problem, Phelps et al., 2012.5 described a way for embedding 3-D radiology models in Portable Document Format (PDF). Hence, it is possible to convert 3-D DICOM (Digital Imaging and Communications in Medicine) dataset into a surface representation file and subsequently export this data to MeshLab software (Visual Computing Lab - ISTI - CNR; http://meshlab.sourceorge.net/; version 1.3.3) aiming to create an acrobat-compatible file, which allows converting this file into U3D (Universal 3D file format). This file type can be attached in Adobe Acrobat X Pro (www.adobe.com/products/acrobatpro.html), and posteriorly it can be embebed in PowerPoint presentations (Windows Office 2013) through a plugin called PDF3D (http://www.pdf3d.com/PDF3D_in_PowerPoint.php), which adds 3D PDF interactive views inside PowerPoint slide presentations (Figure 5).

\section{Conclusions}

With 3-D imaging of the maxillofacial skeleton becoming widespread, the use of Microsoft PowerPoint facilitates the presentation and sharing of clinical information with a variety of clinical and non-clinical audiences in a variety of settings. Hence, the present description about guidelines and their functionality could be applied to all versions of Power Point for Windows. However, screen recording and Apps are available only in 2013 Microsoft Power Point version.

Mobile devices, both android and iOS (iPhones and iPads) systems, allow all the Slide Master functionalities. However, these systems show some limitations regarding the applicability of the tools described in the present article. The options "insert photo album", "screen recording" and use of Apps are not able to be used in current versions of PowerPoint for these systems. Similarly, Microsoft PowerPoint version for Mac OS does not provide these tools. A solution for the problem related to the image insertion may be the use of Iphotos ${ }^{\circledR}$ that allows insertion of multiple pictures into the slide.
Regarding the unavailable screen recording option,Apple ${ }^{\circledR}$ offers the QuickTime ${ }^{\circledR}$ as a free downloadable software. Thus, there is an interesting possibility of embedding a DICOM movie (converted from a Quick Time movie) in a customized PowerPoint presentation.

Another relevant issue in Power Point presentations is the security to avoid unauthenticated changes. Since an increasing number of presentations availabe in the World Wide Web has become widespread, a password can be assigned to prevent unauthorized users from opening/ editing a document. Hence, to create a password the option of exporting presentation should be selected, followed by Package Presentation for CD and advanced options to enter a valid password aiming to open or modify a presentation.

Future trends have been heading for making increasingly dynamic and interactive presentations. In this context, a new available tool for any device is the Office Mix that allows to record, write, and draw on slides just like a whiteboard, as well as to add quizzes, video, and interactive content.

In brief, the advent of digital midia improved quickly and more cost effectively the registration of clinical cases. In an educational perspective, Microsoft PowerPoint may facilitate the organization of cases conducted in Oral and Maxillofacial surgical services, enhancing academic activities and scientific knowledge.

\section{References}

Scully C (2005). Oral cancer; the evidence for sexual transmission. Br Dent J, 199, 203-7.

Dantas TS, de Barros Silva PG, Sousa EF, et al (2016). Influence of educational level, stage, and histological type on survival of oral cancer in a Brazilian population: a retrospective study of 10 years observation. Medicine, 95, 2314.

Hegarty D (1999). Presentations with dentofacial planner images. Am J Orthod Dentofacial Orthop, 116, 114-6.

Regennitter FJ (2000). Powering up your PowerPoint presentations. Am J Orthod Dentofacial Orthop, 118, 116-20.

Halazonetis DJ (2002). New features of Powerpoint 2002. Am J Orthod Dentofacial Orthop, 122, 668-72.

Halazonetis DJ (1998). Making slides for orthodontic presentations. Am J Orthod Dentofacial Orthop, 113, 586-9. 